\title{
The SMC X-ray binary SXP4.78 : a new Type II outburst and the identification and study of the optical counterpart
}

\author{
I.M. Monageng ${ }^{1 \star}$, M.J. Coe ${ }^{2}$, L.J. Townsend ${ }^{3}$, D.A.H. Buckley ${ }^{1}$, V.A. McBride ${ }^{1}$, \\ P.D. Roche ${ }^{4}$, J.A. Kennea ${ }^{5}$, A. Udalski ${ }^{6}$, P.A. Evans ${ }^{7}$ \\ ${ }^{1}$ South African Astronomical Observatory, P.O Box 9, Observatory, 7935, Cape Town, South Africa \\ ${ }^{2}$ Physics \& Astronomy, University of Southampton, SO17 1BJ, UK \\ ${ }^{3}$ Department of Astronomy, University of Cape Town, Private Bag X3, Rondebosch 7701, South Africa \\ ${ }^{4}$ School of Physics \&5 Astronomy, Cardiff University, The Parade, Cardiff, CF24 3AA \\ ${ }^{5}$ Department of Astronomy and Astrophysics, The Pennsylvania State University, University Park, PA 16802, USA \\ ${ }^{6}$ Warsaw University Observatory, Al. Ujazdowskie 4, 00-478 Warszawa, Poland \\ ${ }^{7}$ University of Leicester, X-ray and Observational Astronomy Research Group, Leicester Institute for Space and Earth Observation, \\ Department of Physics \& Astronomy, University Road, Leicester LE1 7RH, UK
}

Accepted XXX. Received YYY; in original form ZZZ

\begin{abstract}
SXP4.78 was originally discovered in 2000 as a pulsar in the Small Magellanic Cloud (SMC) by the Rossi X-ray Timing Explorer (RXTE,) but it was not spatially located at that time. A new detection in 2018 with the Neil Gehrels Swift Observatory during a Type II outburst permitted its position to be accurately located and its optical counterpart identified. We report X-ray and optical monitoring covering epochs before and during the outburst. Using photometric data we show the long-term variability of the Be disc where we present flux and colour changes associated with the disc growth and decay over a period of $\sim 6000$ days. We show evidence of disc growth during the recent outburst through an increase in the $\mathrm{H} \alpha$ equivalent width and photometric flux. Period analysis was performed using both optical photometric and spectroscopic data, but with no significant detection of an orbital period. A modest periodic signature of 2.65 days was detected from the OGLE $I$ band data, however, but we attribute that to the non-radial pulsations (NRPs) of the Be star. We also obtained a blue spectrum from the Southern African Large Telescope (SALT) which permits us to classify the spectral type as B0.5 IV-V.
\end{abstract}

Key words: stars: emission line, Be X-rays: binaries

\section{INTRODUCTION}

High mass X-ray binaries (HMXBs) are binary star systems comprising a compact object (neutron star or black hole) and a massive early-type companion (O or B spectral type). Based on the luminosity class of the massive companion HMXBs are generally divided into supergiant X-ray binaries (sgXBs; luminosity class I and II) and Be X-ray binaries (BeXBs; luminosity class III, IV and V). BeXBs, which make up the largest subclass of the HMXB systems (49\% Coleiro \& Chaty 2013), have a companion star with a geometrically thin Keplerian disc and a neutron star (NS; only one black hole system has been confirmed to date; Casares et al. 2014). The disc variability is traced through the variability of the Balmer emission lines in the optical spectra,

^ E-mail: itu@saao.ac.za the strongest and best-studied of which is the $\mathrm{H} \alpha$ line. The $\mathrm{X}$-ray behaviour of BeXBs is characterised by two types of outburst events, namely type I $\left(L \leq 10^{37} \mathrm{erg} . \mathrm{s}^{-1}\right)$ and type II ( $L \geq 10^{37}$ erg.s ${ }^{-1}$; Stella et al. 1986). Type I outbursts occur regularly and are typically separated by the orbital period. The origins of type II outbursts have remained elusive, with several models proposed for them (e.g. Moritani et al. 2013; Martin et al. 2014; Monageng et al. 2017a). A comprehensive review of BeXBs is given in Reig (2011).

SXP 4.78, the subject of this paper, was reported as a new X-ray transient source in the Small Magellanic Cloud (SMC) from observations obtained with the Neil Gehrels Swift Observatory (Gehrels et al. 2004; as Swift J005139.2-721704) when it reached a luminosity of $4 \times$ $10^{37}$ erg.s ${ }^{-1}$ (Coe et al. 2018a). Analysis of NICER and Fermi/GBM data revealed that the source is associated with a known pulsar, XTE J0052-723, with a pulse period of 
$4.78 \mathrm{~s}$ (Strohmayer et al. 2018). XTE J0052-723 was first discovered on 27 Dec 2000 (JD 2451906) during regular RXTE monitoring of the SMC (Corbet et al. 2001; Laycock et al. 2003). During this discovery outburst, there were 7 PCA detections of a strong $4.78 \mathrm{~s}$ periodicity. A set of slew observations were used to localise the source of the pulsations to RA $(\mathrm{J} 2000)=00: 52: 17$, Dec $(\mathrm{J} 2000)=-72: 19: 51$, which we now know to be several arc-minutes away from the actual source position $(\mathrm{RA}=00: 51: 39.2, \mathrm{Dec}=-72: 17: 03.6 \mathrm{Coe}$ et al. 2018a).

The spectrum is described by an absorbed blackbody and power-law model best-fitted by $N_{H}=0.56 \pm 0.04 \times$ $10^{22} \mathrm{~cm}^{-2}, k T=0.120 \pm 0.03 \mathrm{keV}$ and $\Gamma=1.07 \pm 0.03$ (Guillot et al. 2018). The refined position of the pulsar revealed an association with the B-star from Massey's catalogue [M2002] SMC 20671 (Massey 2002), making the source a newly discovered BeXB. Maravelias et al. (2018) performed spectral classification and conclude that the spectral type of the donor is B1-2e. The authors also report the presence of the $\mathrm{H} \alpha$ and $\mathrm{H} \beta$ lines in emission, which have previously been reported to be absent (Evans et al. 2004). Period analysis of OGLE III and IV data showed a strong signal at 1.805 days (Coe et al. 2018b). It was also noted from the OGLE data that the $I$-band magnitude reached its brightest state in $\sim 6000$ days, suggesting a recent growth in the circumstellar disc size associated with the X-ray activity. In this paper we present multiwavelength observations of SXP4.78, where we study its recent behaviour using data from SALT, Swift, OGLE, RXTE and LCO.

\section{OBSERVATIONS}

\subsection{Swift}

The source was discovered by Neil Gehrels Swift Observatory (Gehrels et al. 2004) whilst observing another SMC transient system, SXP 91.1 (Coe et al. 2018a). By good fortune SXP 4.78 lies only $\sim 5$ arcminutes from SXP 91.1 and hence was comfortably in the same XRT field of view. The source was then monitored over the $0.3-10 \mathrm{keV}$ range throughout the outburst. The observations cover a couple of months and typical exposure times used were 0.9-1.1 ks, depending upon the spacecraft visibility window at that time. The XRT lightcurve was produced following the instructions described in the Swift data analysis guide (http://www.swift.ac.uk/analysis/xrt/). The results are shown in Fig. 1.

\subsection{SALT}

We obtained optical spectra of SXP4.78 using the South African Large Telescope (SALT; Buckley et al. 2006). The observations were performed using the Robert Stobie Spectrograph (RSS; Burgh et al. 2003; Kobulnicky et al. 2003) in long slit mode between 16 November 2018 and 23 December 2018. Various grating set-ups were used, covering different wavelength regions: PG2300 (3850 - $4900 \AA)$, PG0900 $(4350-7400 \AA)$ and PG1800 (5985 - 7250 ̊). Table 1 summarises the set-up of the SALT observations. The primary reductions (overscan correction, bias subtraction, gain correction and amplifier cross-talk correction) were performed
Table 1. A summary of the settings for the SALT observations

\begin{tabular}{lcccc}
\hline \hline \multicolumn{5}{c}{ Grating Wavelength Exposure } \\
Grating & angle & range $(\AA)$ & time $(\mathrm{s})$ & Resolution $(\AA)$ \\
\hline PG0900 & 30.5 & $4350-7400$ & 1800 & 6.1 \\
PG1800 & 36.875 & $5985-7250$ & 900 & 1.8 \\
PG2300 & 30.5 & $3850-4900$ & 2000 & 2.1 \\
\hline
\end{tabular}

using the SALT pipeline (Crawford et al. 2012). The remainder of the data reduction process, which consists of identification of the arc lines, background subtraction and extraction of the 1D spectra was undertaken using various tasks in $\mathrm{IRAF}^{1}$. Fig 2 shows an example of the blue spectrum with all the line species shown.

\subsection{OGLE}

The OGLE project (Udalski et al. 1997, 2015) provides long term I-band photometry with a cadence of 1-3 days. Once the optical counterpart of the source had been identified through the accurate Swift XRT position with the Be star [M2002] SMC 20671, it was possible to retrieve over 17 years of photometric monitoring in the I-band. The source is identified as SMC 719.21.22049 in OGLE IV and SMC 101.4.21932 in OGLE III. The whole lightcurve of the $I$-band magnitudes is shown in Fig. 3 and reveals 2-3 major optical peaks over the period of the OGLE observations - the most recent being coincident with the current X-ray outburst. A detailed presentation of the current outburst may be seen in Fig. 1.

\section{$2.4 \quad$ LCO}

CCD imaging photometry was obtained through the Las Cumbres Observatory (LCO) global network of robotically controlled telescopes, which currently consists of two $2 \mathrm{~m}$, nine $1 \mathrm{~m}$, and ten $0.4 \mathrm{~m}$ instruments, distributed amongst six sites covering both hemispheres (Brown et al. 2013; Boroson et al. 2014). The optical imaging instrumentation (cameras and filters) on telescopes in each aperture class are identical, providing consistent datasets over long timescales (years). The data is all processed using the BANZAI automatic pipeline (McCully et al. 2018), which performs instrumental signature removal (bad pixel masking, bias and dark removal, flat-field correction), astrometric fitting and source catalogue extraction. In this case observations were initially obtained from the $2 \mathrm{~m}$ Faulkes Telescope South at Siding Spring, Australia, in the B (20 s exposure), V (10 s), R $(10 \mathrm{~s})$ and I (10 s) bands using the Spectral imaging system. Follow-up monitoring observations were then conducted using the LCO $1 \mathrm{~m}$ facilities at Siding Spring and CTIO, Chile, in the SDSS g $\mathrm{g}^{\prime}(20-30 \mathrm{~s}), \mathrm{r}^{\prime}(10-20 \mathrm{~s})$ and i' (10-20 s) bands using the Sinistro imaging system. The LCO observations for our programme span a period between 19 October 2018 and 13 December 2018.

1 Image Reduction and Analysis Facility: iraf.noao.edu 

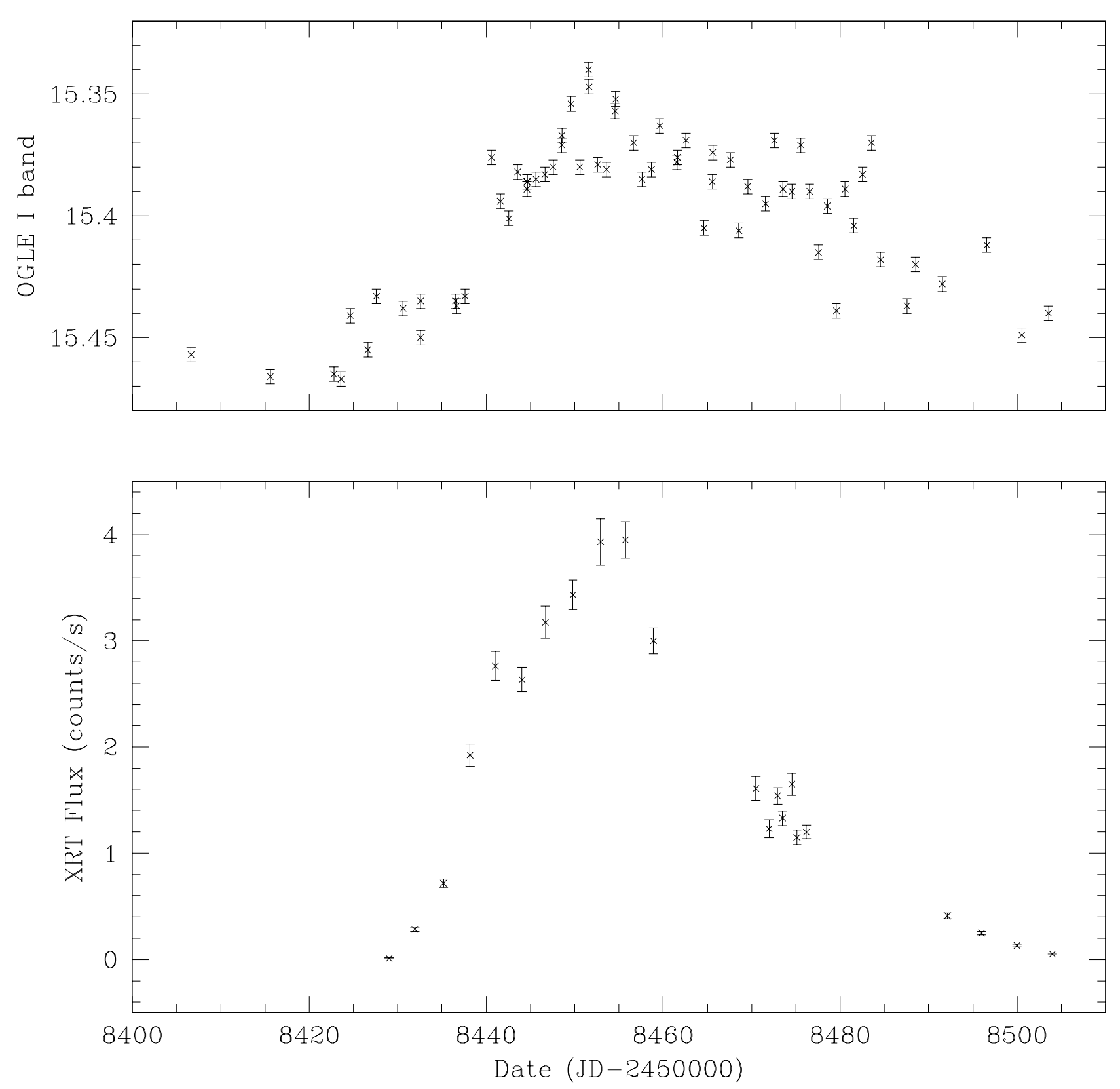

Figure 1. The $I$-band and X-ray measurements of SXP 4.78 during the current outburst. The OGLE data are shown in the top panel and the Swift X-ray measurements are in the lower panel.

\subsection{RXTE}

Once the association of XTE J0052-723 and Swift J005139721704 had been confirmed, we searched the RXTE archive for historical detections of the source. We found no detections of the source in addition to the two outbursts published in the literature (Laycock et al. 2003; Galache et al. 2008. The discovery outburst peaked at roughly $10^{38} \mathrm{erg} / \mathrm{s}$ and lasted for at least 5 weeks (Laycock et al. 2003). The peak luminosity was derived from RXTE spectra, so should be used as a rough estimate only, though Laycock et al. (2003) made attempts to account for any non-pulsed emission from other sources in the field of view. RXTE measured the spin period of the system 5 times during the outburst, as shown in Table 2 and originally listed in Laycock et al. (2003).

Galache et al. (2008) show evidence for one other notable detection of the source around MJD 53725. This outburst lasted for around 50 days and had a similar peak luminosity to the discovery outburst. Some additional detections are noted in Klus et al. (2014), but these are likely spuri- 


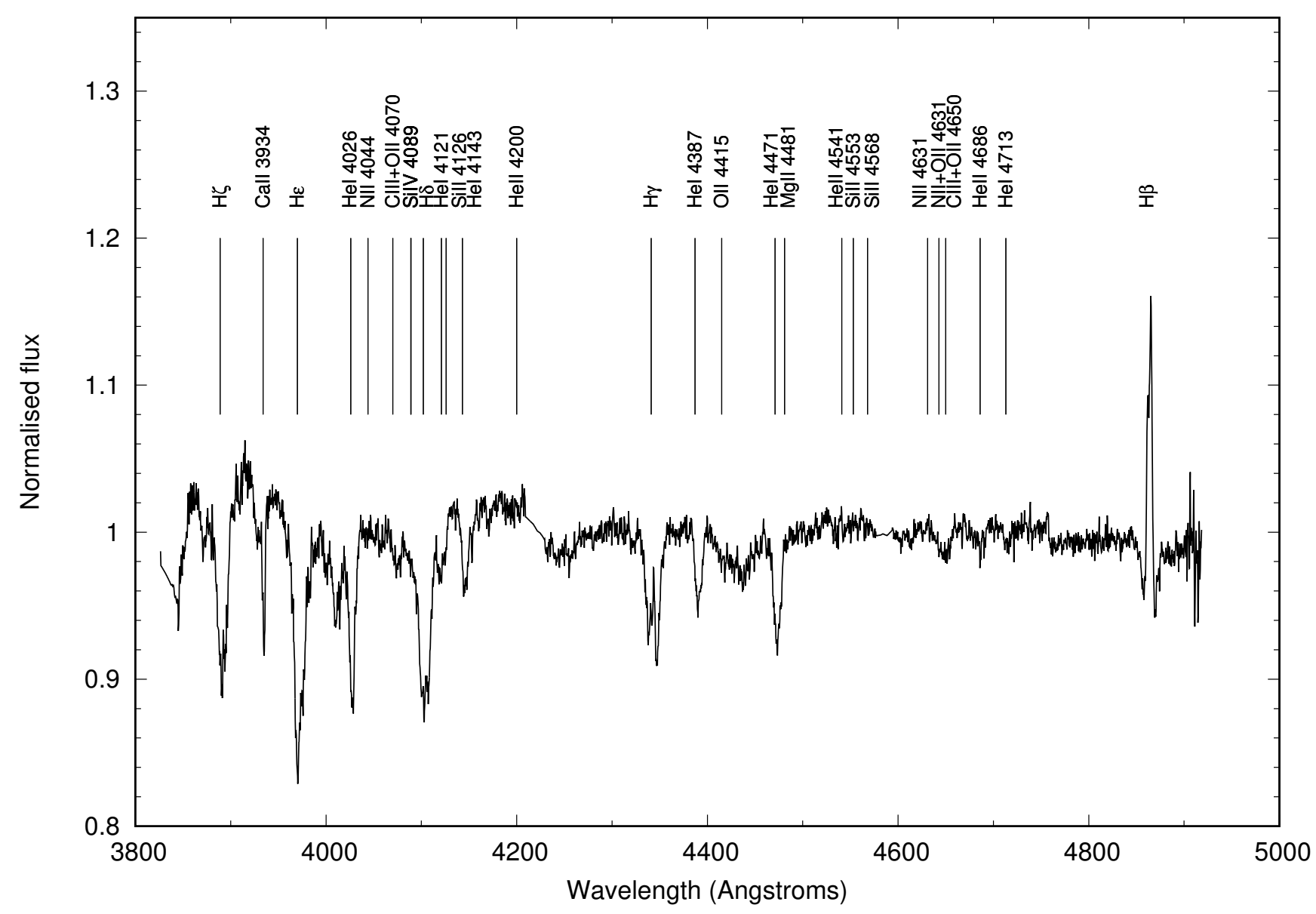

Figure 2. An example SALT blue spectrum of SXP4.78 with all the line species labelled.

ous events in the automated period detection routine used in the search for pulsars in RXTE data (see Galache et al. 2008 for methods), as they are of lower significance and result in periods significantly different from the two bright archival outbursts and the current outburst.

There seems to be little evidence for periodic repetition in the three outbursts recorded for this source. There are roughly 1820 days and 4700 days between the first and second outburst and second and current outburst respectively. Yang et al. (2017) report that the source was in the field of view of RXTE around 800 times, only being detected 11 times. The source was not detected by any other X-ray telescope until the current outburst, despite the deep XMM and Chandra surveys of the SMC.

\section{RESULTS \& DISCUSSION}

\subsection{Search for the binary period}

\subsubsection{OGLE data}

It is well-known that many Be/X-ray binary systems reveal evidence for a binary modulation in the OGLE $I$-band data (Bird et al. 2012). It is believed that this optical signature arises from the disruption of the circumstellar disk close to the time of the periastron passage of the neutron star.
Table 2. RXTE spin period history, except the last value which is from the 2018-19 outburst obtained with NuSTAR (Laycock et al. 2003; Antoniou et al. 2018)

\begin{tabular}{ccc}
\hline \hline MJD & Spin period (s) & Error (s) \\
\hline 51905 & 4.7817 & 0.0001 \\
51912 & 4.7818 & 0.0001 \\
51918 & 4.782 & 0.001 \\
51927 & 4.7820 & 0.0001 \\
51933 & 4.7823 & 0.0001 \\
53725 & 4.78126 & 0.00009 \\
53733 & 4.78149 & 0.00015 \\
53738 & 4.78149 & 0.00007 \\
53747 & 4.78172 & 0.00007 \\
53754 & 4.78172 & 0.00012 \\
53774 & 4.78172 & 0.00013 \\
58439 & 4.781607 & 0.000007 \\
\hline
\end{tabular}

Since SXP 4.78 has been monitored by the OGLE project for over 17 years (see Fig. 3), we searched these data for a binary signature. It is clear from that figure that the $I$-band has peaked 2-3 times over this time frame, the most recent peak correlating with the X-ray outburst discussed in this paper.

The Corbet diagram (Corbet 1984) shows a general correlation between pulse and binary periods and hence provides a useful guide to the most probable time domain to 


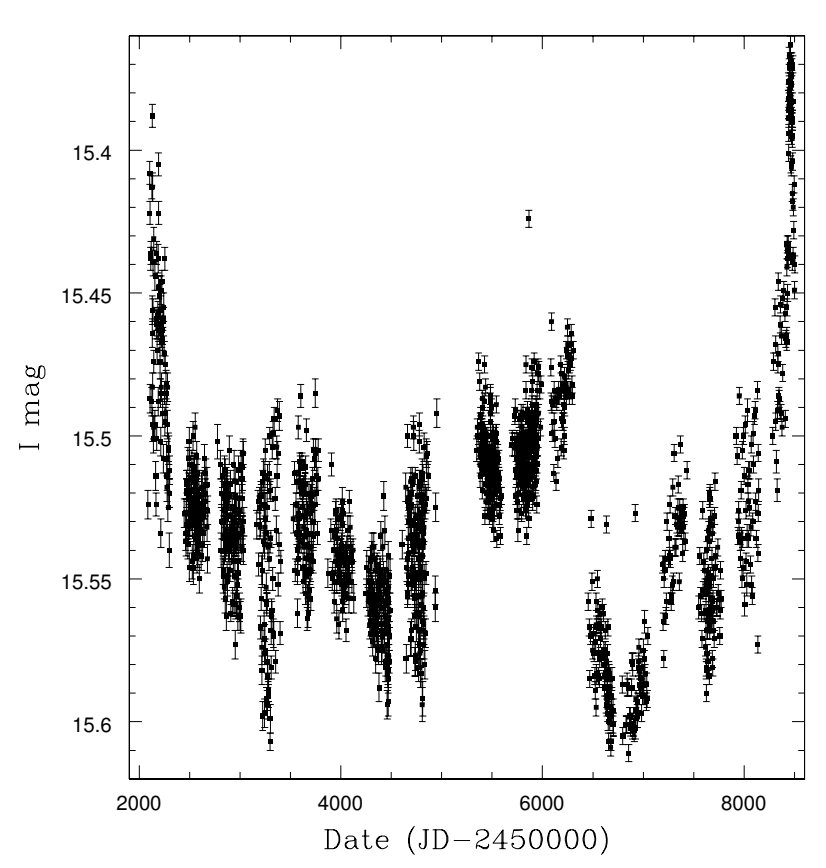

Figure 3. The 17 years of OGLE $I$-band data from OGLE III and IV.
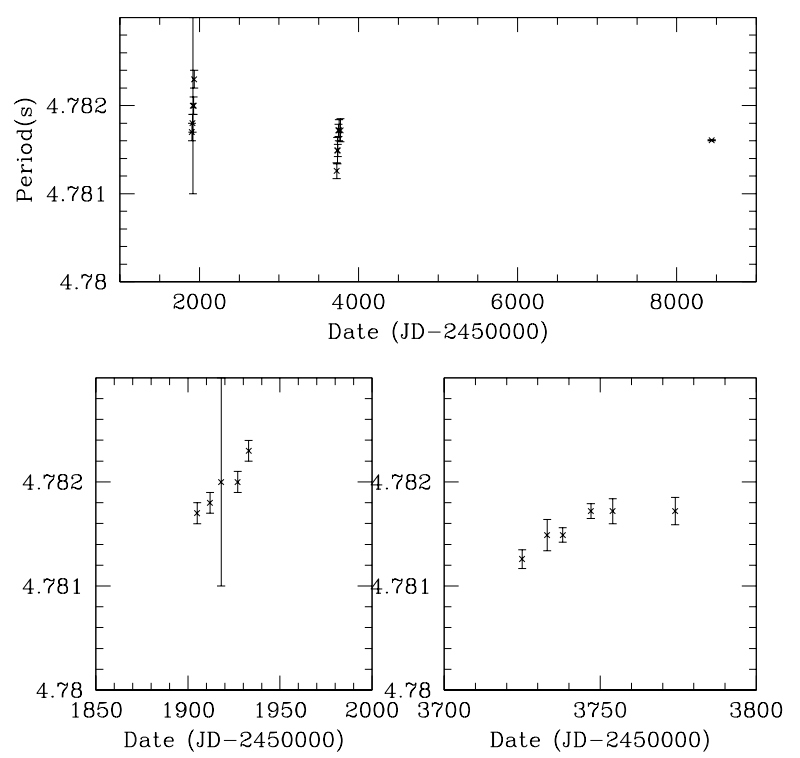

Figure 4. (Top panel) Published spin period history of SXP4.78. All points except the last one are from RXTE; the last one comes from NICER - see text for references. (Lower 2 panels) The details of the spin period evolution as seen by RXTE during two previous outbursts.

search. In the case of SXP 4.78 it is the range is $20-50$ days, though the search was extended to cover all periods from $2 \mathrm{~d}$ to $300 \mathrm{~d}$. The data were de-trended using a $3 \mathrm{rd}$ order polynomial and a Lomb-Scargle routine was used for the search, but no significant peak was found at any time over the whole search range. In addition, smaller subsets of the OGLE data were studied (for example the most recent 2-3 years), but no evidence for a significant coherent periodic signature was found. The largest peak seen at any time corresponded to a period of $2.65 \mathrm{~d}$ but that is most likely related to non-radial pulsation (NRP) behaviour in the Be star. Certainly when the data are folded at that period the resulting profile is sinusoidal in nature, consistent with such an explanation.

\subsubsection{RXTE data}

The remarkable stability of the pulse period of SXP4.78 is evident in Table 2 and Fig. 4, and continues to be so during the current outburst (Antoniou et al. 2018). It is clear from Fig. 3 that the discovery outburst coincided with a previous period of enhanced disk emission (the only other time the optical flux of this source has been recorded above 15.4 magnitudes in the $I$-band). Interestingly, the second Xray outburst around MJD 53725 coincides with a period of low optical flux and hence likely a smaller disk. This is yet another example of X-ray outbursts occurring in Be/X-ray binaries when the circumstellar disk appears to be small. In other instances where such behaviour was observed the implication of the small disc size (traced through the $\mathrm{H} \alpha$ EW) was suggested to be due to a precessing warped disc, where the low values of the $\mathrm{H} \alpha \mathrm{EW}$ are due to geometric effects (Moritani et al. 2013). Another possible reason for this could be the presence of an eccentric disc with the elongation part pointing away from the observer (Martin et al. 2014; Monageng et al. 2017a).

It is unusual to observe a NS spinning down during a period of enhanced accretion. Fig. 4 clearly shows this is the case for both previous outbursts of this source. It is difficult to say from these data whether the cause of the spin-down is due to accretion torques (in which case the torque would be acting in the opposite direction to the spin of the NS), or whether it is due to Doppler shifting. If the latter, we can constrain the orbital period to greater than roughly 100 days as this is twice the length of the longest outburst, in which we only see the spin period increase. Thus we are not seeing the corresponding decrease from motion on the other side of the orbit. This is slightly longer than suggested by the Corbet diagram, but certainly possible. The period changes are likely due to a combination of both factors, making constraining the orbital period difficult.

\subsubsection{Radial velocity search}

We performed radial velocity (RV) analysis using the blue spectra taken with the PG2300 grating. This was done using cross-correlation, by applying the iterative procedure described in Foellmi et al. (2003), Manick et al. (2015) and Monageng et al. (2017b) to create a high signal-to-noise template, which is summarised as follows:

- After the spectra were corrected for the heliocenter, they were normalised and then the continuum level was subtracted.

- The spectra were then converted to a logarithmic wavelength scale and then sorted on the basis of their signal-tonoise ratio. 
Table 3. A summary of the RV measurements of SXP4.78

\begin{tabular}{cc}
\hline \hline MJD & Radial velocity $\left(\mathrm{km} \mathrm{s}^{-1}\right)$ \\
\hline 58438.8267593 & $144 \pm 11$ \\
58449.8244676 & $137.7 \pm 8.9$ \\
58452.8029398 & $122.3 \pm 7.4$ \\
58453.7979977 & $134.7 \pm 8.1$ \\
58454.8230093 & $144.9 \pm 4.9$ \\
58456.8003588 & $136.0 \pm 8.9$ \\
58457.8088773 & $163 \pm 11$ \\
58457.8325231 & $139.6 \pm 9.3$ \\
\hline
\end{tabular}

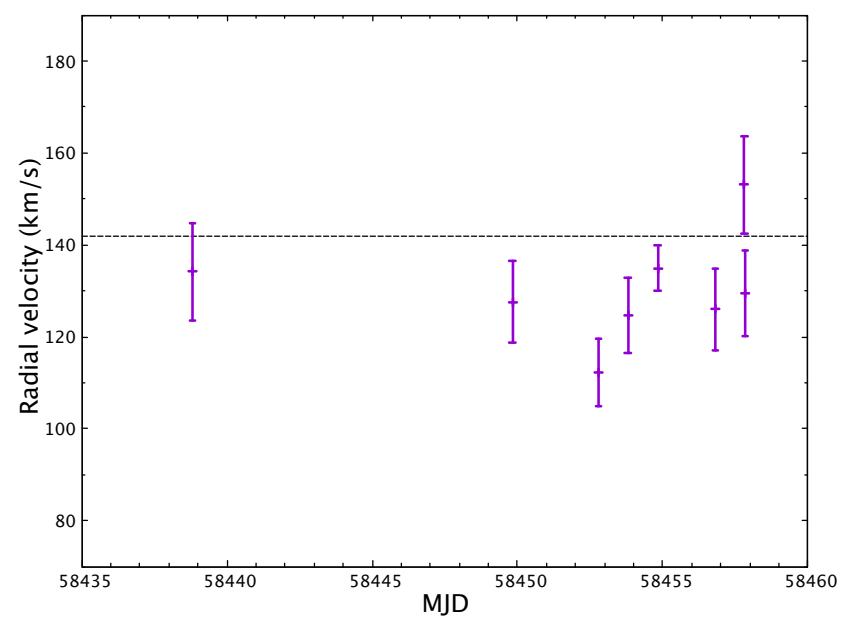

Figure 5. The measured radial velocities of SXP4.78. The dashed line shows the recessional velocity of the SMC.

- The spectrum with the highest signal-to-noise ratio was then used as template in the first iteration of crosscorrelation.

- The spectra were then shifted to the same wavelength as the template spectrum using the results of the first crosscorrelation iteration.

- The spectra were then combined to create a high signalto-noise spectrum to be used as a template for the final cross-correlation iteration.

Table 3 is a summary of the RV shifts. Due to the infilling present in the Balmer lines, we performed crosscorrelation analysis considering only the metal lines. Different combinations of these line species were considered. The analysis was also performed using all the lines available in the blue spectra with little differences in the overall variability. Fig. 5 shows the RV measurements from the whole wavelength range. The measured RVs shown here display little evidence of any periodicity, but rather show an average velocity consistent with that of the redshift velocity of the SMC (McConnachie 2012). We note that the semi-amplitude is expected to be very low (on the order of $20 \mathrm{~km} / \mathrm{s}$ ), considering the spectral type of the primary (see section 3.2 ) and a lower limit of the orbital period of 20 days, making it difficult to detect any periodicity with our poor sampling and relatively large error bars of the RVs.
Table 4. Magnitude range for the different filters obtained from observations reported in this work.

\begin{tabular}{|c|c|}
\hline Filte & magnitude range \\
\hline$I$ & $15.6-15.3$ \\
\hline$V$ & $15.7-15.5$ \\
\hline$B$ & $15.8-15.6$ \\
\hline$g^{\prime}$ & $15.6-15.5$ \\
\hline$r^{\prime}$ & $15.6-15.7$ \\
\hline$i^{\prime}$ & $15.8-15.7$ \\
\hline
\end{tabular}

\subsection{Spectral classification}

The blue spectrum in Fig. 2 shows the sum of 8 observations with a combined averaged exposure of $1800 \mathrm{~s}$. The spectrum is clearly an early type star with many of the Balmer lines showing infilling, likely from the circumstellar disc emission. The CIII+OII blend at 4650 constrains the spectral type to earlier than B3 (Evans et al. 2004) and the weak He II 4686 further constrains the spectral type to B0.5. Most of the luminosity indicators at this spectral type involve metallic lines species, so we use the observed magnitude $(V-15.59)$ together with the distance modulus of the SMC (18.95, Graczyk et al. 2013) to constrain the luminosity class to a IV-V star. The spectral type is slightly earlier that that of both Maravelias (Maravelias et al. 2018) and Evans (Evans et al. 2004) due to our significantly increased exposure. This enabled detection of a very faint HeII 4686 line.

\subsection{The CMD}

A colour-magnitude diagram (CMD) of the $8.6 \times 8.6$ arcmin region around SXP 4.78 was produced based on the OGLEIII photometric maps of the SMC (Udalski et al. 2008). The result is shown is shown in Fig. 6. The red dot indicates the position of SXP 4.78. It is worth noting that though the star is not located on the SMC main sequence its position is consistent with other Be/X-ray binary systems in the SMC. Seven such sources were chosen at random and are indicated by blue triangles. The comparison sources used were SXP 7.78, SXP 9.13, SXP 15.3, SXP 31.0, SXP 91.1, SXP 264 and SXP 293. Details of these comparison sources may be found in Coe \& Kirk (2015). Assuming a distance modulus of 18.95 to the SMC (Graczyk et al. 2013) and a reddening of E(B-V) $=0.08$ (Schwering \& Israel 1991) a B1V star should exhibit an observed (V-I) colour of -0.018 . The colour reported here is $(\mathrm{V}-\mathrm{I}) \sim+0.1$ indicating considerable local reddening. It is highly likely that in all SXP systems the excess reddening is caused by local absorption associated with the Be star's circumstellar disk and wind outflow.

\subsection{Optical colour changes}

Figure 7 shows the long-term evolution of the $(V-I)$ colour index from OGLE, where we see relatively large amplitude changes attributed to the Be disc variability. Figure 8 shows the $(V-I)-I$ colour magnitude diagram, where a positive correlation is seen, i.e. the system reddens as it increases in brightness. Such behaviour has been previously observed in several systems where the variability is explained by a geometric effect resulting from a low viewing angle of the 


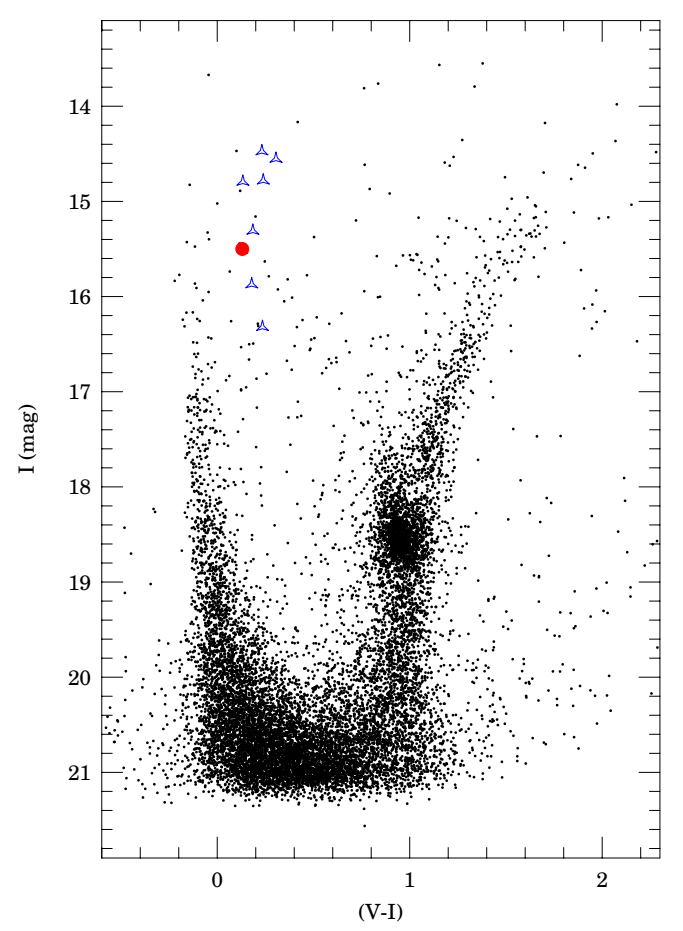

Figure 6. The location of SXP 4.78 on an OGLE CMD. The location of SXP 4.78 is shown by the red circle. Also shown are the location of seven of the Be/X-ray binary systems in the SMC by blue triangles (see text for list).

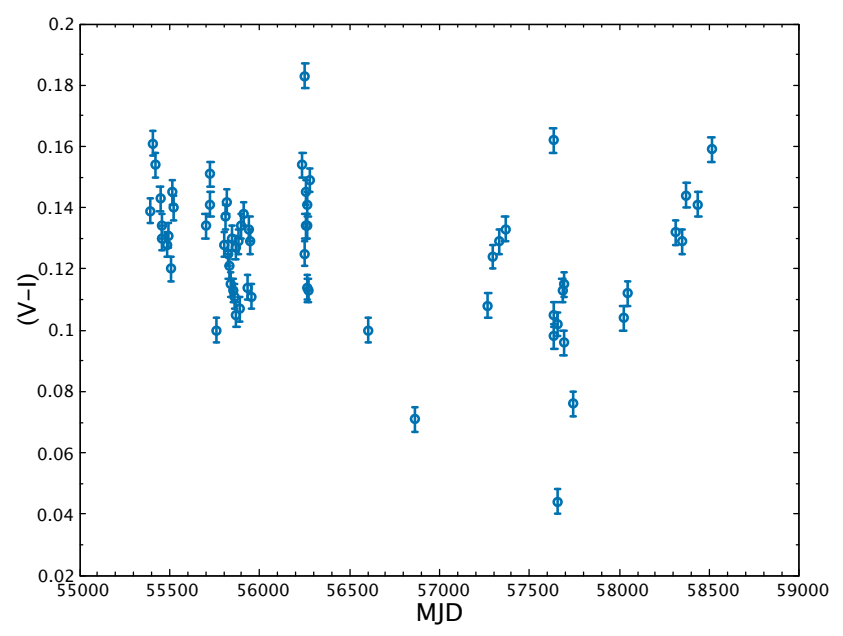

Figure 7. Long-term evolution of the $(V-I)$ colour index - data from the OGLE project.

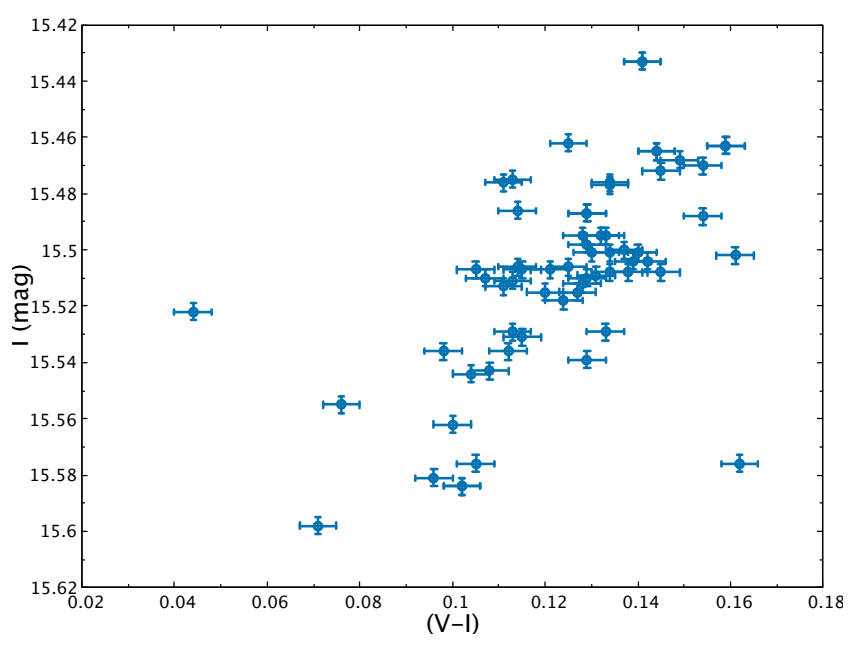

Figure 8. $(V-I)-I$ colour magnitude diagram.

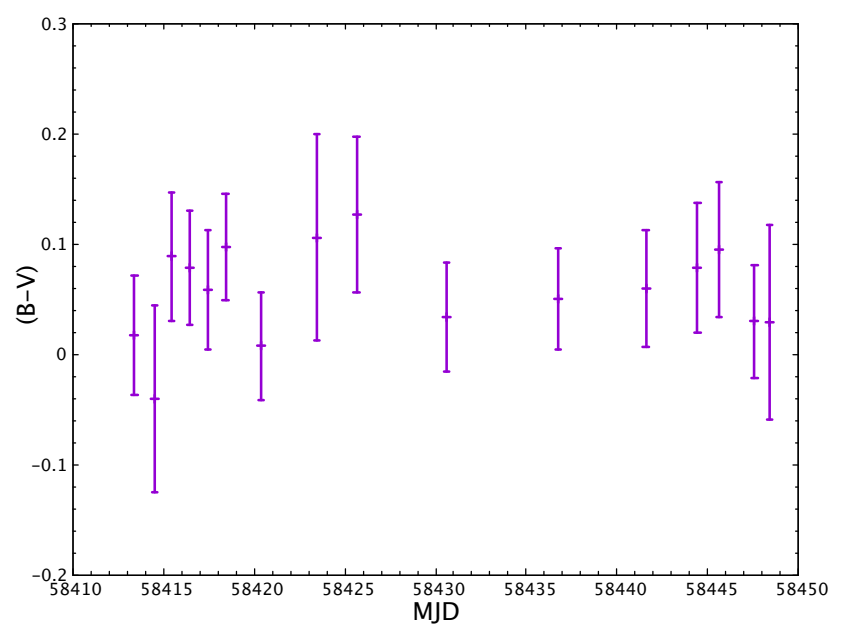

Figure 9. Evolution of the $(B-V)$ colour index during the recent Type II outburst. Data from LCO observations.

Be disc relative to the observer which causes an increase in brightness and red continuum as the radius of the disc increases (Harmanec 1983; Rajoelimanana et al. 2011; Reig \& Fabregat 2015). The suggestion of a low inclination of the Be disc is corroborated by the $\mathrm{H} \alpha$ emission line having a singlepeaked morphology in all our observations (see section 3.5). Figure 9 displays the LCO $(B-V)$ colour index for observations taken during the rise of the recent outburst where no significant variability in the short-term measurements is seen.

\subsection{Be disc variability}

In addition to the blue spectra, we have obtained spectra covering the red end. The $\mathrm{H} \alpha$ line is present in emission and shows a single-peaked profile in all our spectra (Fig. 10 is an example of the $\mathrm{H} \alpha$ emission line profile), suggesting the presence of the circumstellar disc throughout our period of monitoring. The $\mathrm{H} \alpha$ equivalent width (EW) is used as an indicator of the size of the disc in Be stars. Fig. 11 shows the 
Table 5. H $\alpha$ equivalent width measurements of SXP4.78

\begin{tabular}{ccc}
\hline \hline MJD & EW $(\AA)$ & Grating \\
\hline 58436.7981482 & $10.09 \pm 0.28$ & PG1800 \\
58436.7945139 & $11.24 \pm 0.39$ & PG1800 \\
58438.8108333 & $10.84 \pm 0.82$ & PG900 \\
58438.8002662 & $9.86 \pm 0.37$ & PG900 \\
58466.8024421 & $13.00 \pm 0.19$ & PG1800 \\
58464.8145486 & $12.19 \pm 0.59$ & PG1800 \\
58465.8335648 & $12.37 \pm 0.70$ & PG1800 \\
58467.8104398 & $11.86 \pm 0.21$ & PG1800 \\
58436.7981482 & $10.09 \pm 0.28$ & PG1800 \\
58436.7945139 & $11.24 \pm 0.39$ & PG1800 \\
58472.8146412 & $12.74 \pm 0.66$ & PG1800 \\
58473.7998611 & $13.27 \pm 0.24$ & PG1800 \\
58474.7845833 & $12.88 \pm 0.72$ & PG1800 \\
58475.7893171 & $13.40 \pm 0.77$ & PG1800 \\
\hline
\end{tabular}

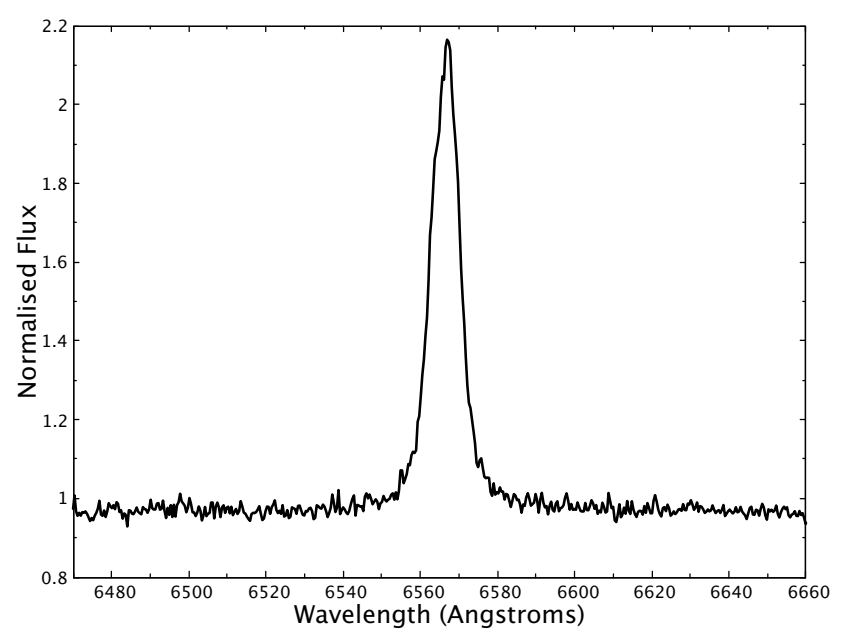

Figure 10. An example of the $\mathrm{H} \alpha$ emission line in SXP4.78

evolution of the EW, where the recent measurements (58466 $<$ MJD $<58475)$ have shown a slight increase from those at the start of the monitoring campaign $(58436<$ MJD $<$ 58438 ), suggesting disc growth in this period. The measured EW values are given in Table 5. Fig. 11 also shows the OGLE $I$-band lightcurve overplotted with the EW measurements, and we note that the gradual increase in the $I$-band flux until it reaches a high state matches the EW behaviour, further supporting the suggestion of disc growth. We note that Evans et al. (2004) report the $\mathrm{H} \alpha$ line in absorption from previous observations. Reig et al. (1997) reported the existence of a correlation between the maximum $\mathrm{H} \alpha \mathrm{EW}$ and orbital period in BeXBs. From a rough interpolation from that relationship and using the maximum value of the $\mathrm{H} \alpha$ EW measured in this work $(13.40 \AA)$, this results in an orbital period of $\sim 25$ days. This is in good agreement with the range of orbital periods suggested based on the Corbet diagram (see section 3.1.1).

\section{CONCLUSION}

In this work we have presented X-ray and optical analysis of SXP4.78 studying its historic and recent behaviour. We have shown that the Be disc has undergone long-term variability

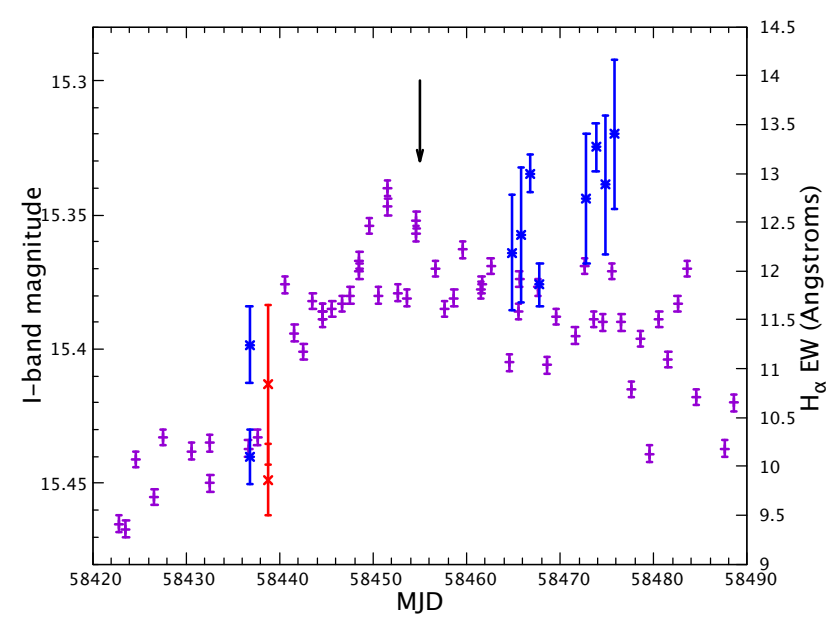

Figure 11. OGLE $I$-band light curve (purple circles) and $\mathrm{H} \alpha$ EW measurements from PG900 (red squares) and PG1800 (blue triangles) gratings. The arrow indicates the epoch of the X-ray outburst peak.

through the analysis of photometric flux and colour changes. We conducted period searches using both photometric and spectroscopic data with no significant detections of any orbital modulation. A detection of a period at 2.65 days was made from the long-term OGLE $I$-band data which is most likely associated with the NRPs of the Be star. The orbital period remains elusive at this time. We have shown that the circumstellar disc has exhibited recent growth through the increase in optical magnitude and $\mathrm{H} \alpha \mathrm{EW}$. We believe that it is this disc growth that has supplied matter resulting in the Type II outburst. Our blue spectrum from SALT permits us to classify the spectral type as B0.5 IV-V, typical for an optical counterpart to a Be/X-ray binary system.

\section{ACKNOWLEDGEMENTS}

The OGLE project has received funding from the National Science Centre, Poland, grant MAESTRO 2014/14/A/ST9/00121 to AU. DAHB, LJT and IMM are supported by the South African NRF. Some of the observations reported in this paper were obtained with the Southern African Large Telescope (SALT), as part of the Large Science Programme on transients 2018-2-LSP-001 (PI: Buckley). Polish participation in SALT is funded by grant no. MNiSW DIR/WK/2016/07. This work makes use of observations from the LCOGT network. This work is based on the research supported by the National Research Foundation of South Africa (Grant numbers 98969 and 93405).

\section{REFERENCES}

Antoniou V., Zezas A., Hong J., Kennea J., Tomsick J., Haberl F., 2018, The Astronomer's Telegram, 12234

Bird A. J., Coe M. J., McBride V. A., Udalski A., 2012, MNRAS, 423, 3663

Boroson T., et al., 2014, in Observatory Operations: Strategies, Processes, and Systems V. p. 91491E, doi:10.1117/12.2054776 Brown T. M., et al., 2013, PASP, 125, 1031 
Buckley D. A. H., Swart G. P., Meiring J. G., 2006, in Society of Photo-Optical Instrumentation Engineers (SPIE) Conference Series. p. 62670Z, doi:10.1117/12.673750

Burgh E. B., Nordsieck K. H., Kobulnicky H. A., Williams T. B., O'Donoghue D., Smith M. P., Percival J. W., 2003, in Iye M., Moorwood A. F. M., eds, Proc. SPIEVol. 4841, Instrument Design and Performance for Optical/Infrared Ground-based Telescopes. pp 1463-1471, doi:10.1117/12.460312

Casares J., Negueruela I., Ribó M., Ribas I., Paredes J. M., Herrero A., Simón-Díaz S., 2014, Nature, 505, 378

Coe M. J., Kirk J., 2015, MNRAS, 452, 969

Coe M. J., Kennea J. A., Buckley D., Udalski A., 2018a, The Astronomer's Telegram, 12209

Coe M. J., Kennea J. A., Buckley D., McBride V., Udalski A., Evans P., Roche P., Townsend L., 2018b, The Astronomer's Telegram, 12229

Coleiro A., Chaty S., 2013, ApJ, 764, 185

Corbet R. H. D., 1984, A\&A, 141, 91

Corbet R., Marshall F. E., Markwardt C. B., 2001, IAU Circ., 7562

Crawford S. M., et al., 2012, PySALT: SALT science pipeline, Astrophysics Source Code Library (ascl:1207.010)

Evans C. J., Lennon D. J., Walborn N. R., Trundle C., Rix S. A., 2004, PASP, 116, 909

Foellmi C., Moffat A. F. J., Guerrero M. A., 2003, MNRAS, 338, 360

Galache J. L., Corbet R. H. D., Coe M. J., Laycock S., Schurch M. P. E., Markwardt C., Marshall F. E., Lochner J., 2008, ApJS, 177, 189

Gehrels N., et al., 2004, ApJ, 611, 1005

Graczyk D., Pietrzyński G., Pilecki B., Thompson I. B., Gieren W., Konorski P., Udalski A., Soszyński I., 2013, in de Grijs R., ed., IAU Symposium Vol. 289, Advancing the Physics of Cosmic Distances. pp 222-225 (arXiv:1311.1270), doi:10.1017/S1743921312021436

Guillot S., et al., 2018, The Astronomer's Telegram, 12219

Harmanec P., 1983, Hvar Observatory Bulletin, 7, 55

Klus H., Ho W. C. G., Coe M. J., Corbet R. H. D., Townsend L. J., 2014, MNRAS, 437, 3863

Kobulnicky H. A., Nordsieck K. H., Burgh E. B., Smith M. P., Percival J. W., Williams T. B., O'Donoghue D., 2003, in Iye M., Moorwood A. F. M., eds, Proc. SPIEVol. 4841, Instrument Design and Performance for Optical/Infrared Groundbased Telescopes. pp 1634-1644, doi:10.1117/12.460315

Laycock S., Corbet R. H. D., Coe M. J., Marshall F. E., Markwardt C., Edge W., 2003, MNRAS, 339, 435

Manick R., Miszalski B., McBride V., 2015, MNRAS, 448, 1789

Maravelias G., Antoniou V., Zezas A., Strantzalis A., Hatzidimitriou D., Haberl F., 2018, The Astronomer's Telegram, 12224

Martin R. G., Nixon C., Armitage P. J., Lubow S. H., Price D. J., 2014, ApJ, 790, L34

Massey P., 2002, ApJS, 141, 81

McConnachie A. W., 2012, AJ, 144, 4

McCully C., Volgenau N. H., Harbeck D.-R., Lister T. A., Saunders E. S., Turner M. L., Siiverd R. J., Bowman M., 2018, in Software and Cyberinfrastructure for Astronomy V. p. 107070K (arXiv:1811.04163), doi:10.1117/12.2314340

Monageng I. M., McBride V. A., Coe M. J., Steele I. A., Reig P., 2017a, MNRAS, 464, 572

Monageng I. M., McBride V. A., Townsend L. J., Kniazev A. Y., Mohamed S., Böttcher M., 2017b, ApJ, 847, 68

Moritani Y., et al., 2013, PASJ, 65, 83

Rajoelimanana A. F., Charles P. A., Udalski A., 2011, MNRAS, 413,1600

Reig P., 2011, Ap\&SS, 332, 1

Reig P., Fabregat J., 2015, A\&A, 574, A33

Reig P., Fabregat J., Coe M. J., 1997, A\&A, 322, 193

Schwering P. B. W., Israel F. P., 1991, A\&A, 246, 231
Stella L., White N. E., Rosner R., 1986, ApJ, 308, 669

Strohmayer T. E., et al., 2018, The Astronomer's Telegram, 12222

Udalski A., Kubiak M., Szymanski M., 1997, Acta Astron., 47, 319

Udalski A., et al., 2008, Acta Astron., 58, 329

Udalski A., Szymański M. K., Szymański G., 2015, Acta Astron., 65,1

Yang J., Laycock S. G. T., Christodoulou D. M., Fingerman S., Coe M. J., Drake J. J., 2017, ApJ, 839, 119 